\title{
Scanning and Transmission Electron Microscopy of the Tegument of Paranaella luquei Kohn, Baptista-Farias \& Cohen, 2000 (Microcotylidae, Monogenea), Parasite of a Brazilian Catfish, Hypostomus regani
}

\begin{abstract}
SC Cohen ${ }^{+}$, A Kohn ${ }^{++}$, MFD Baptista-Farias
Laboratório de Helmintos Parasitos de Peixes, Departamento de Helmintologia, Instituto Oswaldo Cruz-Fiocruz, Av. Brasil 4365, 21045-900 Rio de Janeiro, RJ, Brasil

The surface topography and ultrastructure of the tegument of Paranaella luquei Kohn, BaptistaFarias \& Cohen, 2000, a microcotylid monogenean parasite from the gills of Hypostomus regani (Ihering, 1905) (Loricariidae) was studied by scanning (SEM) and transmission electron microscopy (TEM). By SEM, it was observed that the tegument presents transversal ridges, forming folds in the ventral and dorsal surfaces and microvillous-like tegumental projections in the anterior and median regions of body. These projections were also observed by TEM. The tegument is made up of a syncytium delimited by apical and basal plasma membranes, containing inclusion bodies and mitochondria, connected to the nucleated region by means of cytoplasmatic processes. The tegumental cells present a well developed nucleus and cytoplasm containing inclusion bodies, similar to those found on the external layer, mitochondria, rough endoplasmatic reticulum and free ribossomes.
\end{abstract}

Key words: ultrastructure - tegument - Monogenea - Paranaella luquei - catfish

Monogeneans are parasites mainly from the gills of fishes and may exhibit both host and site specificity. Studies made by scanning electron microscopy (SEM) revealed that they present characteristic features which indicate phylogenetic isolation of this group within parasitic Platyhelminthes. Nevertheless, different species of Monogenea may present ultrastructural variations of the tegument, such as presence of microvilli and secretory pores and a wide variation on morphological aspect on surface modifications and sensory structures (Smyth \& Halton 1983). Therefore, it is important to investigate the tegument of each individual species in order to elucidate questions on maintenance and survival of the parasite on their microhabitat.

In Microcotylidae, the tegument of Atriaster sp. and Microcotyle sp. have been studied by transmission electron microscopy (TEM) (Justine 1992, Justine \& Bonami 1993). SEM studies of this fam-

\footnotetext{
${ }^{+}$Corresponding author. Fax: +55-21.260-4866. E-mail: scohen@ioc.fiocruz.br

${ }^{++}$Research fellow I-A from Conselho Nacional de Desenvolvimento Científico e Tecnológico.

Received 24 May 2000

Accepted 20 December 2000
}

ily involved the tegument of $M$. labracis by Oliver (1981), M. sebastis by Alyousif (1986), Metamicrocotyla macracantha by Kohn et al. (1994) and A. heterodus by Santos et al. (1996) and the clamps of M. donavini by Shaw (1979).

The ultrastructure of the spermatogenesis and spermiogenesis of Microcotyle sp., parasite of Hypostomus regani, Brazilian catfish found on the reservoir of the Itaipu Hydroelectric Power Station was studied by Baptista-Farias et al. (1999). This species corresponds to the present species, Paranaella luquei Kohn, Baptista-Farias \& Cohen, 2000.

The purpose of this study was to investigate and describe the surface topography and ultrastructure of the tegument of $P$. luquei from the gills of Hypostomus regani (Ihering, 1905) (Loricariidae).

\section{MATERIALS AND METHODS}

Parasites collected from the gills of Hypostomus regani (Ihering, 1905) (Loricariidae) collected from the reservoir of the Itaipu Hydroelectric Power Station, State of Paraná, Brazil, were fixed in 2.5\% phosphate buffered glutaraldehyde and pos-fixed for $1 \mathrm{~h}$ in $1 \%$ osmium tetroxide in the same buffer. Specimens were dehydrated through an increased graded ethanol series. For SEM study, the material was critical point dried and coated with gold. The observations were made using a Zeiss DSM 940. For TEM study, the material was embedded in Epon. Ultrathin sections were obtained using a Reichert 
Ultracut E-microtome, collected in 200 mesh grids, contrasted with uranyl acetate for $30 \mathrm{~min}$ and lead citrate for 5 min (Reynolds 1963) and observed under a Zeiss EM 900 electron microscope.

\section{RESULTS}

Scanning electron microscopy - The body of $P$. luque $i$ is elongated, with a posterior haptor (Fig. 1). The buccal cavity and the opening of the genital atrium lie in the anterior part of the ventral surface (Fig. 2).

The tegument of the body presents transversal ridges, at regular intervals, forming folds in the ventral and dorsal surfaces (Fig. 2). It presents microvillous-like tegumental projections, covering both ridges and folds. Numerous pores were observed in the folds of the tegument. Pores were sometimes located in the protuberances (Fig. 3). Isolated, dome-shaped, non-ciliated papillae were observed irregularly distributed all over the body surface (Fig. 3).

The haptor is separated from the body proper at the posterior region. It is asymmetrical and triangular and composed of two symmetrical rows of clamps, formed by two opposable hinged jaws and covered by a thin tegument. The internal margins of the clamps are serrated and, depending on state of contraction, may be opened or closed (Fig. 4). The haptoral tegument is similar to the rest of the body, differing only in the fact that the microvillous-like projections are absent (Fig. 5). The tegument around the opening of genital atrium is folded radially, and contains microvillous-like projections and non-ciliated sensory papillae (Fig. 6).

Transmission electron microscopy - Ultrastructural examination of the tegument of $P$. luquei reveals a syncytium delimited by apical and basal plasma membranes (Fig. 7). The apical plasma membrane includes an external coat. Immediately beneath this membrane, the cytoplasm is more electron-dense and fibrous, forming a region that can be interpretated as a terminal web (Fig. 8). The microvillous-like projections observed by SEM are present in the external syncytial layer (Fig. 9).

The tegumental syncytium consists of a slightly granular matrix, containing two types of inclusions: electron-dense bodies, such as secretory granules, membrane-bound, moderately electron-dense, spherical to elongate and electron-lucent vesicles: relatively large, predominant, not distributed homogeneously in the external syncytial layer (Fig. 7). In some regions, these electron-lucent vesicles seem to fuse into a multivesicular body. Ocasionally, electron-lucent vesicles were continuous with the apical plasma membrane. Rounded mitochondria with few cristae were also observed in the external syncytial layer (Fig. 7).
The basal plasma membrane has thin projections into the external layer. The basal lamina lies immediately beneath the basal plasma membrane and has a uniform thickness and numerous deep folds (Fig. 7).

The well-developed musculature is made up of alternating layers of circular and longitudinal nonstriated muscle fibers, formed by both thin and thick filaments. These fibers are separated from each other by interstitial material and are bounded by the sarcolemma. Oval to elongate mitochondria, arranged in groups, were observed associated with the muscle fibers. Some of longitudinal bundles appear more electron-dense than others, possibly due to the contraction of the musculature (Fig. 7).

Tegumental cells, located deep in the parenchyma, are connected to external layer by means of cytoplasmic processes perpendicular to body surface. Because these processes are scarse and not straight, it was not possible to obtain a longitudinal section of them.

Irregularly shaped tegumental cells contains a well-developed nucleus, that occupies a large portion of the cell. Each nucleus has a well defined region of heterochromatin. The cytoplasm contains electron-lucent vesicles, similar to those found in the external syncytial layer (Fig. 10). Mitochondria were observed near the nucleus and are smaller than those of the muscular layer (Fig. 11). Golgi complex and free ribossome, filling the space between organelles and inclusions, were also observed (Fig. 12).

\section{DISCUSSION}

Ultrastructural study of the tegument of Monogenea by SEM had revealed that the topography of the surface shows variations in the surface architecture, most of them with functional significance.

In our specimens, microvillous-like tegumental projections were observed in anterior and middle regions of body. These structures are characteristic of the tegument of the majority of the species studied, including Diclidophora merlangi (by Halton 1979), M. labracis (by Oliver 1981), Heterapta chorinemi (by Ramasamy \& Hanna 1986b). However, they are absent in others, such as Pseudothoracocotyla indica and Bicotyle vellavoli (by Ramasamy \& Hanna 1985, 1986a). The ridges and microvillous-like projections present in the tegument increase the surface area, suggesting metabolic exchange (Oliver 1981) and absorption of micromolecular nutrients from the surrounding environment (Ramasamy \& Hanna 1986b). Halton (1978) suggested that the occurence of microvilli and the absorptive function of the tegument provide evidence of a close 

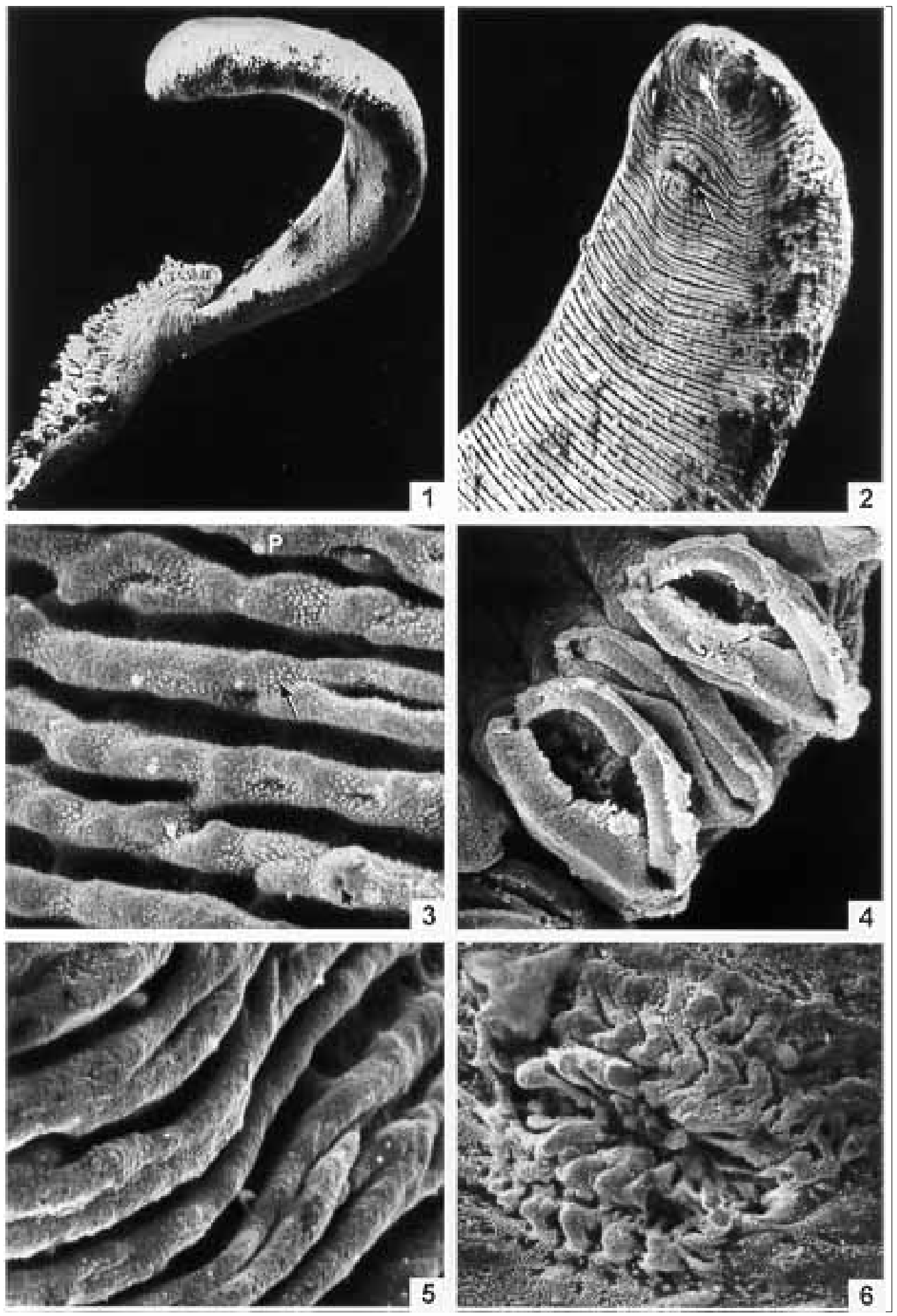

Scanning electron micrographs of Paranaella luquei - Fig.1: total, 120 x. Fig. 2: anterior region of body with subterminal buccal cavity (white arrow) and the opening of genital atrium (black arrow), 330 x. Fig. 3: tegument of median region showing the microvillous-like tegumental projections (arrow), pores (arrowhead) and sensory papillae (P), 3,900 x. Fig. 4: clamps, 1,100 x. Fig. 5: tegument of posterior region of body, devoid of microvillous-like projections, 2,800 x. Fig. 6: genital atrium, 1,300 x 
phylogenetic relationship between Monogenea and Cestoda.

The microvillous-like structures were observed by SEM in other species of Microcotylidae, as in M. labracis (Oliver 1981). Santos et al. (1996) referred these structures as filaments in A. heterodus.
The distribution of microvillous-like projections in the tegument of $P$. luquei is uniform on the ventral and dorsal surfaces and over the ridges and folds of the anterior and middle regions of body. These structures were not observed on the haptoral surface. This finding agrees with the sug-
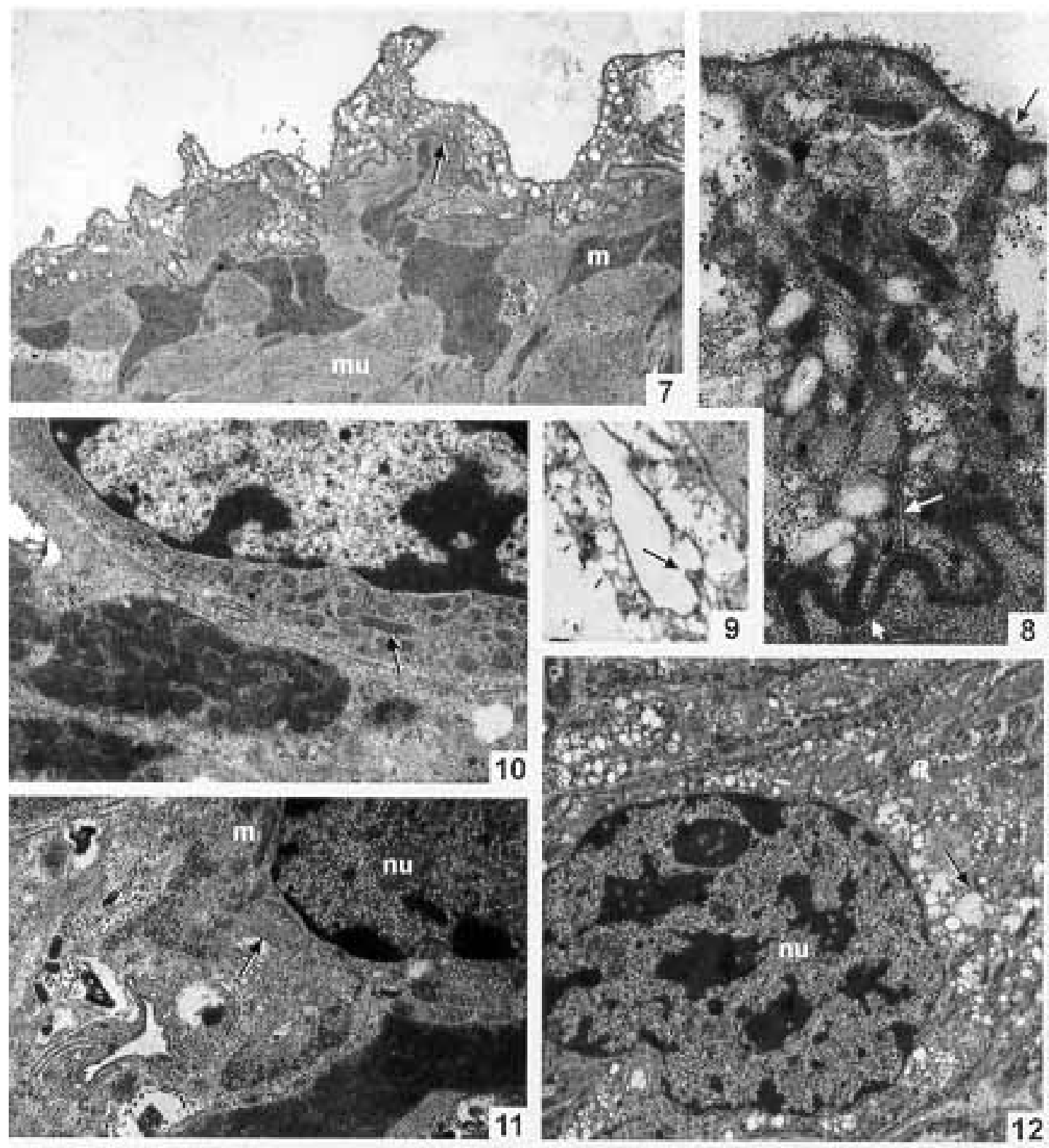

Transmission electron micrographs of the tegument of Paranaella luquei - Fig. 7: transversal section through tegumental fold, showing the external syncytial layer with inclusions, basal lamina (arrow) and muscular layer, constituted of circular and longitudinal fibers $(\mathrm{mu})$ and groups of mitochondria $(\mathrm{m}), 3,700 \mathrm{x}$. Fig. 8: high magnification of the external syncytial layer, showing the terminal web (black arrow) and inclusions; basal plasma membrane with thin projections (white arrow)and basal lamina (arrowhead), 24,000 x. Fig. 9: high magnification of the apical region showing tegumental folds and microvillous-like projections (arrow), 10,000 x. Fig. 10: transversal section through a tegumental cell, showing the cytoplasm with mitochondria (arrow) at the nucleus periphery, 11,000 x. Fig. 11: section of a tegumental cell showing nucleus (nu), mitochondria (m) and Golgi complex, (arrow) 11,500 x. Fig. 12: detail of a tegumental cell with prominent nucleus (nu) and cytoplasm with electronlucent vesicles (arrow) and free ribosomes (R), 5,000 x 
gestion of a close contact with the host tissue for attachment in the gill filaments. The microvillouslike projections, if present, may cause friction in the host tissue. Any increase of the surface area in this region is obtained by pits and depressions. The presence of numerous pores on the tegument of $P$. luquei may indicate that the exocrine discharge occurs by means of this pores, as suggested for Polystoma integerrimum by Williams and McKenzie (1995).

The dome-shaped, non-ciliated papillae observed in the tegument of $P$. luquei are similar of those described for other species, such as $B$. vellavoli (Ramasamy \& Hanna 1986a) and in Allodiscocotyla diacanthi (Ramasamy et al. 1995).

The tegument of $P$. luquei is similar to that described for other Monogenea studied to date. It is made up of an external anucleate layer containing organelles and inclusions, that is in cytoplasmatic continuity with a nucleated region situated beneath the musculature (Smyth \& Halton 1983, Ramasamy et al. 1987, Ramasamy \& Bhuvaneswari 1993). In this respect, the tegument of Monogenea is similar to that of Digenea and Cestoda.

The folds of the apical plasma membrane associated with microvillous-like projections were described in Gotocotyla bivaginalis by Ramasamy and Bhuvaneswari (1993) and agree to the aspect observed by SEM. Those projections may increase the surface area for selective uptake of exogenous material like oxygen and nutrients, and may serve in the secretion of endogenous material and osmorregulation (Ramasamy \& Bhuvaneswari 1993).

The presence of empty vesicles in the apical surface of $P$. luquei suggests a secretory activity (exocrine discharge) or a role in the maintenance of the glycocalyx. The products of exocrine discharge may be incorporated into the glycocalyx forming a mucous layer over the apical plasma membrane. This layer is important in order to minimize the mechanical, ionic, osmotic and immunological damage as observed in D. merlangi (Morris \& Halton 1971), Pricea multae and Vallisia indica (Ramasamy et al. 1986, 1987), and G. bivaginalis (Ramasamy \& Bhuvaneswari 1993).

The basal invaginations observed in the tegument of $P$. luquei were also found in other Monogenea (Morris \& Halton 1971, Brennan \& Ramasamy 1996). The presence of these invaginations suggests the involvement of the tegument in the uptake of nutrients, excretion and osmorregulation (Brennan \& Ramasamy 1996).

The presence of mitochondria in the external syncytial layer may be explained by the necessity of energy for concentration and transport of nutrients.
Beneath the apical plasma membrane of the tegument of $P$. luquei, there was a terminal web made up of microfilaments. This web was also observed in Microcotyle sp. (Justine \& Bonami 1993) and Atriaster sp. (Justine 1992). According to Lyons (1973), the terminal web is characteristic of Polyopisthocotylea and has not been observed in Monopisthocotylea. Justine (1992) suggested that this character was of phylogenetic value.

The synthesis of body inclusions involves the rough endoplasmatic reticulum and Golgi complex, both characteristic organelles of the tegumental cells of $P$. luquei. This suggests that these body inclusions are produced in the tegumental cells and pass up to the external syncytial layer.

\section{ACKNOWLEDGMENTS}

To the Superintendência de Meio Ambiente Aquático, Centro de Pesquisas and to Dr Carla Canzi from Itaipu Binacional for the facilities offered to examine the fish from the reservoir of the Hydroelectric Power Station; to Dr Ortrud Monika Barth from Departamento de Virologia, Instituto Oswaldo Cruz, for the facilities offered for the use of the scanning and transmission electron microscopies.

\section{REFERENCES}

Alyousif MS 1986. Surface topography of Microcotyle sebastis (Trematoda: Monogenea) by scanning electron microscopy. Ann Zool (Agra) 24: 1-7.

Baptista-Farias MFD, Kohn A, Cohen SC 1999. Spermatogenesis and spermiogenesis in Microcotyle sp. (Microcotylidae, Monogenea). J Parasitol 85: 832838.

Brennan GP, Ramasamy P 1996. Ultrastructure of the surface structures and electron immunogold labelling of peptide immunoreactivity in the nervous system of Pseudothoracocotyla indica (Polyopisthocotylea: Monogenea). Parasitol Res 82: 638646.

Halton DW 1978. Trans-tegumental absorption of alfaalanine and alfa-leucine by a monogenean Diclidophora merlangi. Parasitology 76: 29-37.

Halton DW 1979. The surface topography of a monogenean, Diclidophora merlangi, revealed by scanning electron microscopy. Z Parasitenkde 61: 1-12.

Justine JL 1992. Ultrastructure of spermiogenesis, spermatozoa and the tegument in Atriaster sp. (Plathyhelminthes, Monogenea, Polyopisthocotylea, Microcotylidae). Zool Scripta 21: 231-238.

Justine JL, Bonami JR 1993. Virus-like particles in a monogenean (Plathyhelminthes) parasitic in a marine fish. Int J Parasitol 23: 69-75.

Kohn A, Cohen SC, Baptista-Farias MFD 1994. A redescription of the morphology of Metamicrocotyla macracantha (Alexander, 1954) Koratha, 1955 (Monogenea, Microcotylidae) from Mugil liza in Brazil. Syst Parasitol 27: 127-132.

Lyons KM 1973. The epidermis and sense organs of the Monogenea and some related groups. Adv Parasitol 11: 193-232. 
Morris GP, Halton DW 1971. Electron microscope studies of Diclidophora merlangi (Monogenea: Polyopisthocotylea). II. Ultrastructure of the tegument. J Parasitol 57: 59-61.

Oliver G 1981. Etude de Microcotyle labracis Van Beneden et Hesse, 1863 (Monogenea, Polyopisthocotylea, Microcotylidae) au microscope életronique à balayage. Z Parasitenkde 65: 235-240.

Ramasamy P, Bhuvaneswari R 1993. The ultrastructure of the tegument and clamp attachment organ of Gotocotyla bivaginalis (Monogenea: Polyopishtocotylea). Int J Parasitol 23: 213-220.

Ramasamy P, Hanna REB 1985. The surface topography of Pseudothoracocotyla indica (Unnitham, 1956) (Monogenea) from the gills of Scomberomorus commerson. Z Parasitenkde 71: 575581.

Ramasamy P, Hanna REB 1986a. The surface topography of Bicotyle vellavoli (Monogenea) from the gills of Pampus chinensis. Int J Parasitol 16: 591-594.

Ramasamy P, Hanna REB 1986b. The surface topography of a monogenean Heterapta chorinemi from the gills of Scomberoides commersonianus. Int $J$ Parasitol 16: 595-600.

Ramasamy P, Brennan GP, Halton DW 1995. Ultrastructure of the surface structures of Allodiscocotyla diacanthi (Polyopisthocotylea: Monogenea) from the gills of the marine teleost fish, Scomberoides tol. Int J Parasitol 25: 43-54.

Ramasamy P, Hanna REB, Threadgold LT 1986. The surface topography and ultrastructure of the tegument and haptor of Pricea multae (Monogenea). Int J Parasitol 16: 581-589.

Ramasamy P, Hanna REB, Threadgold LT 1987. Scanning and transmission electron studies of the surface of Vallisia indica (Monogenea: Polyopisthocotylea). Int J Parasitol 17: 1187-1195.

Reynolds ES 1963. The use of lead citrate at high $\mathrm{pH}$ as an electron-opaque stain in electron microscopy. $J$ Cell Biol 17: 208-212.

Santos CP, Souto-Padrón T, Lanfredi RM 1996. Atriaster heterodus (Lebedev and Paruchin, 1969) and Polylabris tubicirrus (Paperna and Kohn, 1964) (Monogenea) from Diplodus argenteus (Val., 1830) (Teleostei: Sparidae) from Brazil. J Helminthol Soc Wash 63: 181-187.

Shaw MK 1979. The ultrastructure of the clamp wall of the monogenean gill parasite Gastrocotyle trachuri. $Z$ Parasitenkde 58: 243-258.

Smyth JD, Halton DW 1983. The Physiology of Trematodes, Cambridge University Press, Cambridge.

Williams JB, McKenzie J 1995. Scanning electron microscopy of Polystoma integerrimum (Monogenea, Polystomatidae). Int J Parasitol 25: 335-342. 